SERIES INTRODUCTION

\title{
Stimulation and inhibition of immune responses: an intricate balancing act
}

\author{
Gary Koretzky \\ Department of Pathology and Laboratory Medicine, Signal Transduction Program, Abramson Family Cancer Research Institute, \\ 415 BRBII/III, 421 Curie Boulevard, University of Pennsylvania School of Medicine, Philadelphia, Pennsylvania 19104, USA. \\ Phone: (215) 746-5522; Fax: (215) 746-5525; E-mail: koretzky@mail.med.upenn.edu.
}

J. Clin. Invest. 109:7-8 (2002). DOI:10.1172/JCI200214820.

Development of the adaptive immune system and activation of mature effector lymphocytes require an intricate balance of stimulatory and inhibitory forces. Indeed, as the articles in this Perspective series make clear, the immune system's balancing act must take place simultaneously and in a dynamic manner at multiple levels.

The interplay of positive and negative regulation occurs at each step during lymphocyte activation and, if dysregulated, may have disastrous consequences. Thus, when antigen is first encountered, resting lymphocytes engage ligands via multiple receptors, each with stimulatory or inhibitory potential. The integration of these initial signals ensures that $\mathrm{T}$ cell activation occurs only in the appropriate time and place.

As with the functional opposition between different receptors, many of the biochemical second messenger cascades involved in lymphocyte activation are also mutually antagonistic. For example, kinases and phosphatases regulated by receptor binding may compete for a key residue on an effector molecule, thereby modifying the effector's ability to exert its downstream effect. Interestingly, the function of many of these enzymes may also be dictated by where in the cell they are located, providing yet another level of control to balance the eventual outcome of receptor engagement. Subsequently, when a $\mathrm{T}$ cell is committed to activation, integration of competing signals determines the function of that cell; for example, the pattern of cytokines that will be produced - or even whether that cell will become an active effector or will instead interfere with other stimulated cells. Following the elimination of an antigenic challenge, lymphocyte homeostasis requires that the majority of effector cells generated to combat the pathogen be eliminated. The control of the size of the T cell pool provides another example of a balance of forces. In this response, several receptors that are critical for homeostatic $T$ cell death also play important roles in $T$ cell activation, so the opposition takes place between alternative responses to the ligation of a given receptor.

The tremendous progress in elucidation of the molecular mechanisms underlying the yin and yang of diverse $\mathrm{T}$ cell processes has provided important insights into the basis of immune disorders and clues for the development of novel approaches to manage some of these diseases. In addition, as with most advances in biology, improved understanding of $\mathrm{T}$ cell activation and inactivation has led to a wealth of new questions and arenas for investigation. The seven articles of this Perspective series will highlight advances in our understanding of the balance of signals implicated in $\mathrm{T}$ cell activation, effector function, and apoptosis and will point also to specific issues currently under the most intense study.

\section{Biochemical aspects of the immunological} balancing act

Although none of the components of the T cell antigen receptor (TCR) complex possesses intrinsic enzymatic activity, ligation of the receptor stimulates numerous biochemical second messenger cascades initiated by potent activation of protein tyrosine kinases (PTKs). In fact, PTK activation is a requisite step for essentially all biochemical or functional markers of $\mathrm{T}$ cell activation characterized to date. The first Perspective in this issue of the JCI introduces the signaling events that follow from TCR activation, focusing on the important role of Src family PTKs in initiation of events. Weiss and his coauthors then describe the intricate and complex role played by protein tyrosine phosphatases, in particular CD45, in the regulation of Src PTK function and ultimately the ability of $\mathrm{T}$ cells to become activated. Their article highlights interesting new findings that emphasize how critical structural features of CD45 (and, by extension, probably other protein tyrosine phosphatases) modulate the ability of this enzyme to regulate $T$ cell activation. The authors also consider some of the exciting implications of these observations for human autoimmune disease.

When the structure of the TCR was first elucidated, it emerged that the receptor complex consists not only of variable clonotypic polypeptides derived from rearranging gene segments, but also of noncovalently linked invariant $\mathrm{CD} 3$ chains. Since it was clear that all of the information important for antigen/MHC recognition resides within the clonotypic components of the 
receptor, it was presumed that the $\mathrm{CD} 3$ proteins contribute to intracellular signaling, although no mechanism for this function was yet known. Careful examination of the primary structure of each $\mathrm{CD} 3$ molecule revealed conserved sequences in a unique motif including key tyrosine and leucine/isoleucine residues, later named the immunoreceptor tyrosine-based activation motif (ITAM). In their Perspective, Billadeau and Leibson describe critical features of ITAMs that allow these structures to translate recognition by the TCR of $\mathrm{MHC} /$ antigen complexes into downstream signaling events. The authors go on to articulate what is currently known about the more recently described immunoreceptor tyrosine-based inhibitory motifs (ITIMs), cytoplasmic domains that recruit inhibitory effectors. While comparing and contrasting the biology of ITAM- and ITIM-mediated responses, Billadeau and Leibson emphasize how the signals initiated by engagement of surface receptors are balanced to achieve the desired downstream response.

\section{Ultrastructural organization of signaling \\ in the immune cell}

In addition to activating PTKs, multiple other biochemical signaling cascades are initiated upon engagement of the TCR. One central question in T cell activation is how these myriad pathways are integrated, driving the cell toward the appropriate downstream functions. Tremendous insight into this issue came after the initial description of adapter proteins. Adapters are molecules with no intrinsic effector activity, but which possess interaction domains allowing them to act as scaffolds to build multimolecular complexes. Recent work has revealed the existence of many hematopoietic cell-specific adapters. Later studies, both in cell lines and in vivo, have demonstrated that these proteins are as essential as receptors and enzymes in both the positive and negative regulation of lymphocyte function. In their Perspective, Schraven and colleagues provide examples of cytosolic and transmembrane adapters, indicating how they may function as activators or inhibitors of lymphocyte function. They also describe exciting new findings indicating that diseases severely affecting immune cell function may also be caused by mutations in adapter protein genes.

Over and above the organizing effects of adapter interactions, $\mathrm{T}$ cell activation is also strongly influenced by the topography of the interaction between $\mathrm{T}$ cells and antigen/MHC on antigen-presenting cells. An enormous advance in thinking about this problem came from the realization that the cell surface is not a homogenous unit, but that there are glycolipidenriched islands (lipid rafts or glycolipid-enriched membrane microdomains [GEMs]) within the plasma membrane in which various signaling components may be concentrated. In his Perspective, Dustin applies the notion of lipid rafts to the process of $\mathrm{T}$ cell activation and shows how these microdomains participate in the formation of the immunological synapse, the contact zone between the antigen-presenting cell and the $T$ cell. As Dustin points out, the creation and dissolution of the immunologic synapse, as well as its components, change with cellular activation, providing yet another potential means by which immune cell responses may be modulated for therapeutic advantage.

For decades it was known that productive stimulation of $\mathrm{T}$ cells requires ligation of other receptors in addition to the TCR. In fact, stimulation of the TCR alone leads to long-lived $\mathrm{T}$ cell unresponsiveness and, in some cases, cell death. In keeping with the theme of this series, Thompson's Perspective underscores the notion that costimulatory receptors may serve to promote $\mathrm{T}$ cell function while other costimulatory signals interfere with cellular activation. While much has been learned recently about the biochemical properties of the positive versus negative costimulatory receptors, the Frauwirth and Thompson Perspective points out that the precise mechanisms by which these molecules transduce their signals remain unclear. Nevertheless, manipulation of costimulation will surely represent an important potential modality for future immunotherapies.

\section{Functional modulation of the immunological response}

Once all of the events are in place to initiate a $T$ cell response, further decisions must be made to determine the nature of the ultimate response. In his Perspective, Murphy addresses the important question of how $\mathrm{T}$ cells are instructed or selected to choose the Th1 versus the Th2 fate. This differentiation step is critical, as the cytokines produced by Th 1 and Th 2 cells induce very different host responses. It is clear that cytokines themselves are involved in the cell fate decision, but the point at which cytokines play their pivotal roles remains controversial. As Murphy points out, receptor-generated signals, cytokines, and, ultimately, differential expression of transcription factors all collaborate to determine whether the Th1 or the Th2 response will predominate.

The final Perspective in this series describes events that occur as an immune response is terminated, once the pathogenic challenge has been eliminated. While it is clear that death receptors play a critical role in this process, the Budd Perspective emphasizes that cell death is not all there is to this response. Surprisingly, perhaps, death receptors play critical roles in lymphocyte proliferation as well as in apoptosis, and Budd emphasizes that the context and timing of signaling events, as well as the biochemical cascades themselves, are essential determinants of the ultimate outcome for the cell. Thus, the same signal, delivered at different points in the cell cycle, may lead to dramatically different downstream events.

Collectively, these articles provide a snapshot of our current appreciation for several aspects of lymphocyte differentiation, proliferation, and death. As the receptors and effectors critical for these processes have been characterized, it has become increasingly clear that signals that are stimulatory under certain circumstances may be inhibitory under others, dependent on timing and location. Certainly, it will be necessary to take into account the potential for the same signals to result in either a positive or negative outcome as we consider novel ways to modulate immune cells to treat or prevent human disease. 